\title{
Synthesis and reactivity of small phosphorus-containing dendritic wedges (dendrons)
}

\author{
Laurent Brauge, Valérie Maraval, Régis Laurent, Anne-Marie Caminade,* and Jean- \\ Pierre Majoral*
}

Laboratoire de Chimie de Coordination du CNRS, 205 route de Narbonne, 31077 Toulouse

Cedex 4, France

E-mail: caminade@lcc-toulouse.fr;majoral@lcc-toulouse.fr

\section{Dedicated to Professor Marcial Moreno-Manas on the occasion of his $60^{\text {th }}$ birthday \\ (received 18 Dec 01; accepted 31 Mar 02; published on the web 08 Apr 02)}

\begin{abstract}
The synthesis of two small dendrons (dendritic wedges) is reported and the reactivity of the functionality located at the core is studied. The first dendron has no reactive functionality on the surface, but possesses a vinyl group activated by a $\mathrm{P}=\mathrm{N}-\mathrm{P}=\mathrm{S}$ linkage at the core; this vinyl group reacts slowly but cleanly with 2,3-dimethylbutadiene in Diels-Alder additions. The second dendron has aldehyde groups on the surface and one protected phenol group at the core. The deprotection occurs in very mild conditions, leading to a dendron having one phenol and four aldehyde groups. The reactivity of the phenol is demonstrated by the addition of a diisocyanate, affording a carbamate function and the grafting of an isocyanate group at the core of the dendron.
\end{abstract}

Keywords: Dendron, dendrimer, phosphorus, vinyl, phenol

\section{Introduction}

Dendritic macromolecules are highly branched nanosized compounds having well defined threedimensional size, shape, topology and functionalities. These aesthetic molecules have attracted considerable attention over the past decade, reinforced by promising applications. ${ }^{1}$ The initial compounds in this field were dendrimers, ${ }^{2}$ i.e. dendritic compounds having functionalities only on the surface. However, the usefulness of dendritic wedges (also called dendrons), ${ }^{3}$ having functionalities on the surface and another single functionality at the core was rapidly demonstrated after these pioneering investigations (Figure 1). 

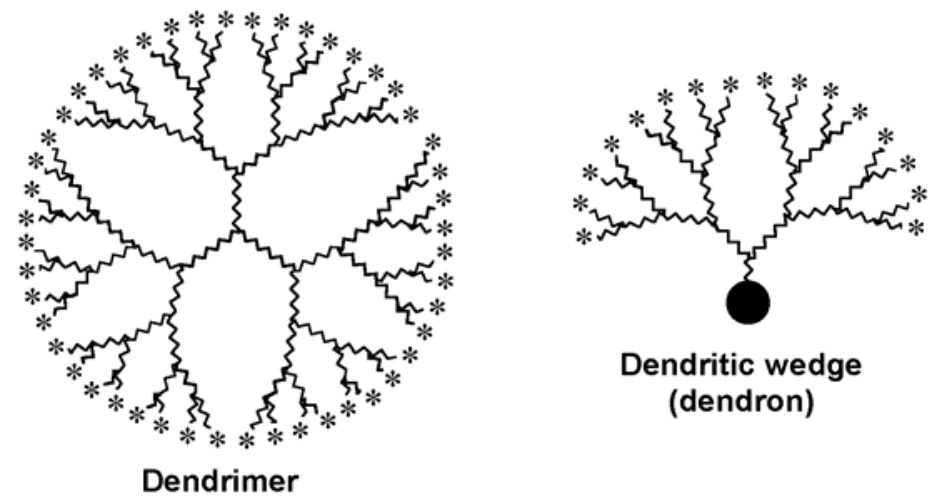

Figure 1. Schematic drawing of a dendrimer and a dendron.

Pursuing our work dealing with the synthesis and study of properties of phosphoruscontaining dendrimers, ${ }^{4}$ we have developed several strategies to functionalize the surface ${ }^{5}$ or even the branches ${ }^{6}$ of dendritic compounds. Recently, we became interested in the synthesis of phosphorus-containing dendrons, ${ }^{7}$ that allowed us to obtain macromolecules having original architectures. The chemical diversity of the functionality located at the core, its reactivity and its compatibility with the functionalities located on the surface remains an interesting problem. We wish to report some examples dealing with the synthesis and reactivity at the core of phosphoruscontaining dendrons, potentially useful building blocks for the synthesis of more complex macromolecules.

\section{Results and Discussion}

We have already demonstrated that a vinyl group activated by a $\mathrm{P}=\mathrm{N}-\mathrm{P}=\mathrm{S}$ linkage located at the core of dendrons possesses an interesting reactivity toward primary and secondary amines. ${ }^{7 \mathrm{a}, 7 \mathrm{c}-7 \mathrm{e}}$ We decided to test another type of reactivity of this group, that is Diels-Alder additions. In order to avoid any side reactions, we utilized a dendron having no reactive functional groups on the

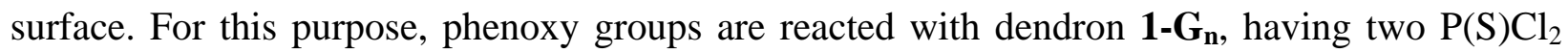

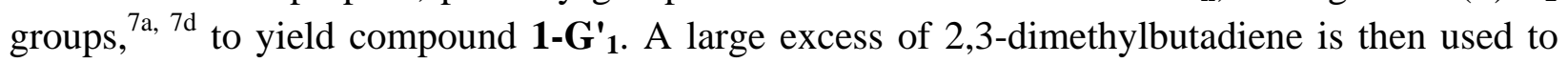
ascertain the reactivity of the vinyl group of dendron $\mathbf{1 - \mathbf { G } _ { \mathbf { 1 } }}$ (Scheme 1 ). The reaction proceeds slowly and necessitates 4 days in toluene at $100^{\circ} \mathrm{C}$ for completion. The reaction is monitored by ${ }^{31} \mathrm{P}$ NMR, which displays in particular the deshielding of the doublet corresponding to the $\mathrm{PPh}_{2}$ group from $9.6 \mathrm{ppm}$ for $\mathbf{1 - \mathbf { G } _ { \mathbf { 1 } }}$ to $19.9 \mathrm{ppm}$ for $\mathbf{2}-\mathbf{G}_{\mathbf{1}}$. Dendron $\mathbf{2}-\mathbf{G}_{\mathbf{1}} \mathbf{1}_{\text {is }}$ isolated in good yield after work up (82\%), but this Diels-Alder reaction is quite slow and necessitates drastic conditions, which could be inapplicable to dendrons having also functionalities on the surface. Thus, it appears that this pathway to functionalize the core of dendrons is not particularly attractive, despite the presence of the $\mathrm{P}=\mathrm{N}-\mathrm{P}=\mathrm{S}$ linkage, for which we have demonstrated a useful reactivity toward electrophiles. ${ }^{6}$ 


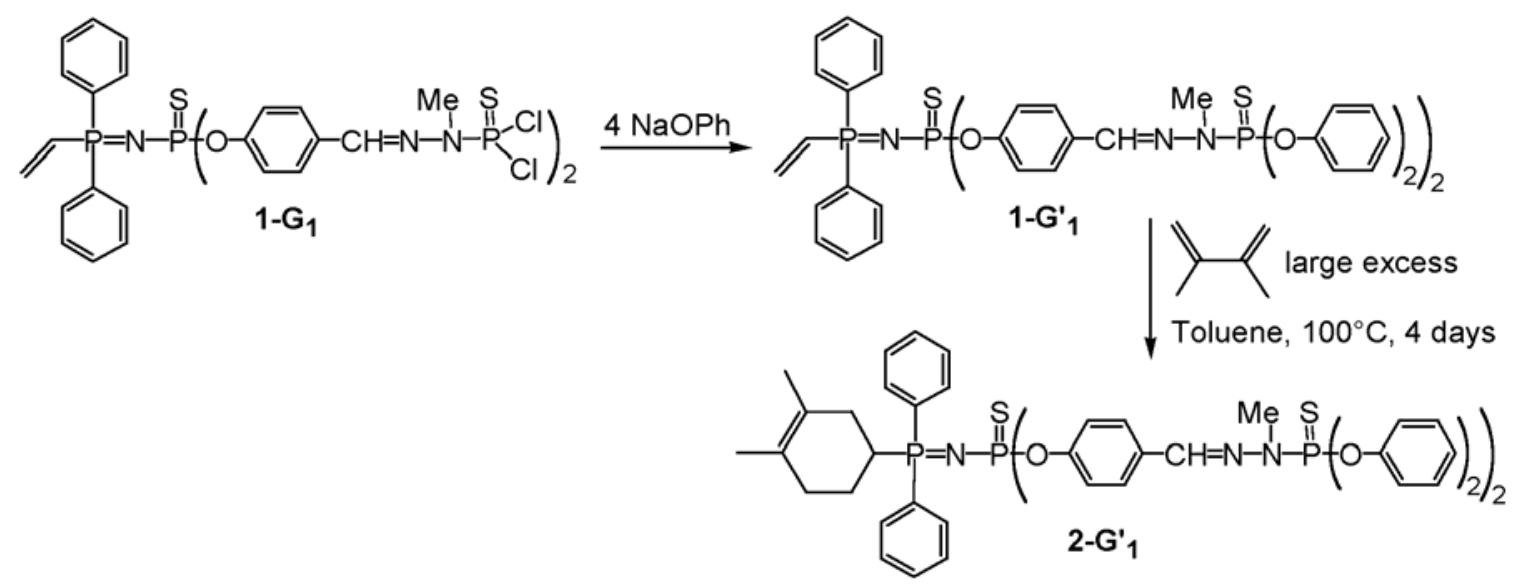

Scheme 1. Synthesis of dendron 1-G' ${ }_{1}$ and Diels-Alder reaction at its core.

Furthermore, it would be desirable to have dendrons possessing a single reactive group at the core to avoid side reactions. For this purpose, we decided to apply our "classical" method of synthesis of phosphorus-containing dendrimers ${ }^{4 \mathrm{a}, 4 \mathrm{~b}, 4 \mathrm{~d}}$ to 4 -acetoxy-benzaldehyde. Indeed, this compound possesses a protected phenol, which could be deprotected in mild conditions after the synthesis of the dendron. The first step of the synthesis is the condensation with $\mathrm{H}_{2} \mathrm{NNMeP}(\mathrm{S}) \mathrm{Cl}_{2}$, the second step is the nucleophilic substitution of $\mathrm{Cl}$ by hydroxybenzaldehyde

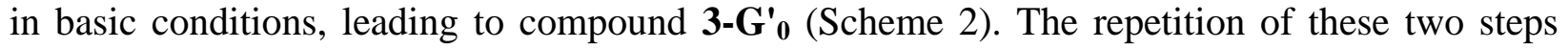
leads finally to the dendron $\mathbf{3}-\mathbf{G}_{\mathbf{1}}$, possessing one ester functionality at the core and four aldehyde groups on the surface. These reactions are monitored by ${ }^{1} \mathrm{H}$ NMR for the condensation reactions (disappearance of the signal corresponding to $\mathrm{CHO}$ ) and by ${ }^{31} \mathrm{P} \mathrm{NMR}$ for the substitution reactions (slight shielding on going from $\mathrm{P}(\mathrm{S}) \mathrm{Cl}_{2}$ to $\mathrm{P}(\mathrm{S})(\mathrm{OAr})_{2}$ ).

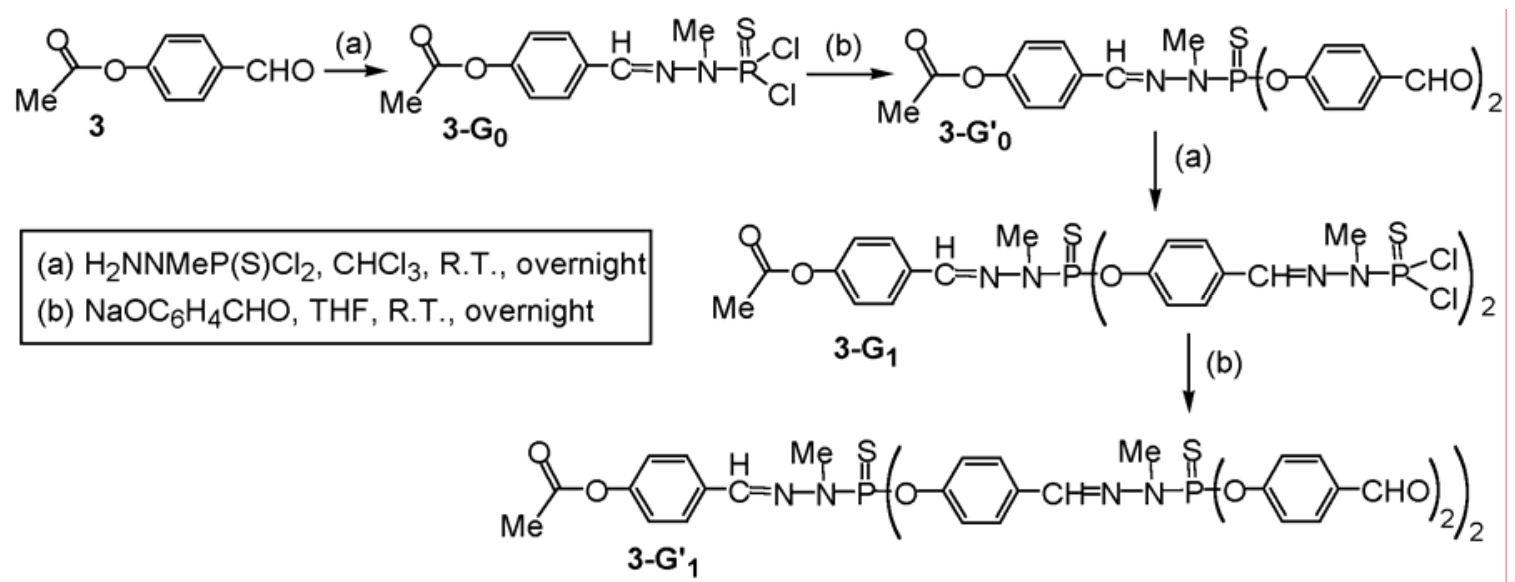

Scheme 2. Synthesis of dendron $\mathbf{3}-\mathbf{G}_{\mathbf{n}}(\mathbf{n}=0,1)$ from the 4-acetoxy-benzaldehyde core. 


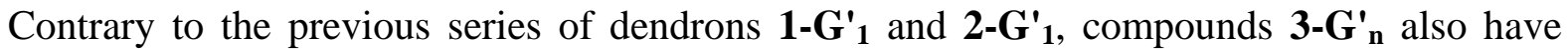
functionalities (aldehydes) on the surface; thus, the reactivity carried out at the core must not interfere with the surface. First, we attempted to deprotect the phenol in a classical way, using a mixture of organic solvents and water or methanol, in the presence of $\mathrm{KOH}$ or $\mathrm{K}_{2} \mathrm{CO}_{3}$. In all cases, the deprotection is only partial and a degradation of the dendron is observed. Consequently, we decided to apply a very mild method, using guanidine as desacetylating agent. ${ }^{8}$ The reaction is almost instantaneous, no side reaction is observed with the aldehydes, but the

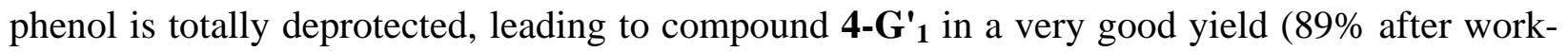
up) (Scheme 3). This compound is characterized by NMR and Mass spectrometry (FAB).

The presence of a phenol group at the core of dendron 4-G' ${ }_{\mathbf{1}}$ offers a number of possibilities for further functionalizations. As an example, an excess (6 equivalents) of 1,6-

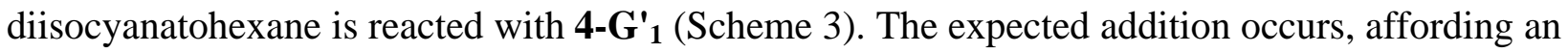
isocyanate core and a carbamate function, characterized in particular by ${ }^{13} \mathrm{C} \mathrm{NMR}$ by the appearance of a singlet at $154.3 \mathbf{p p m}$. Dendron 5-G' ${ }_{\mathbf{1}}$ also is characterized by Mass spectrometry (FAB).

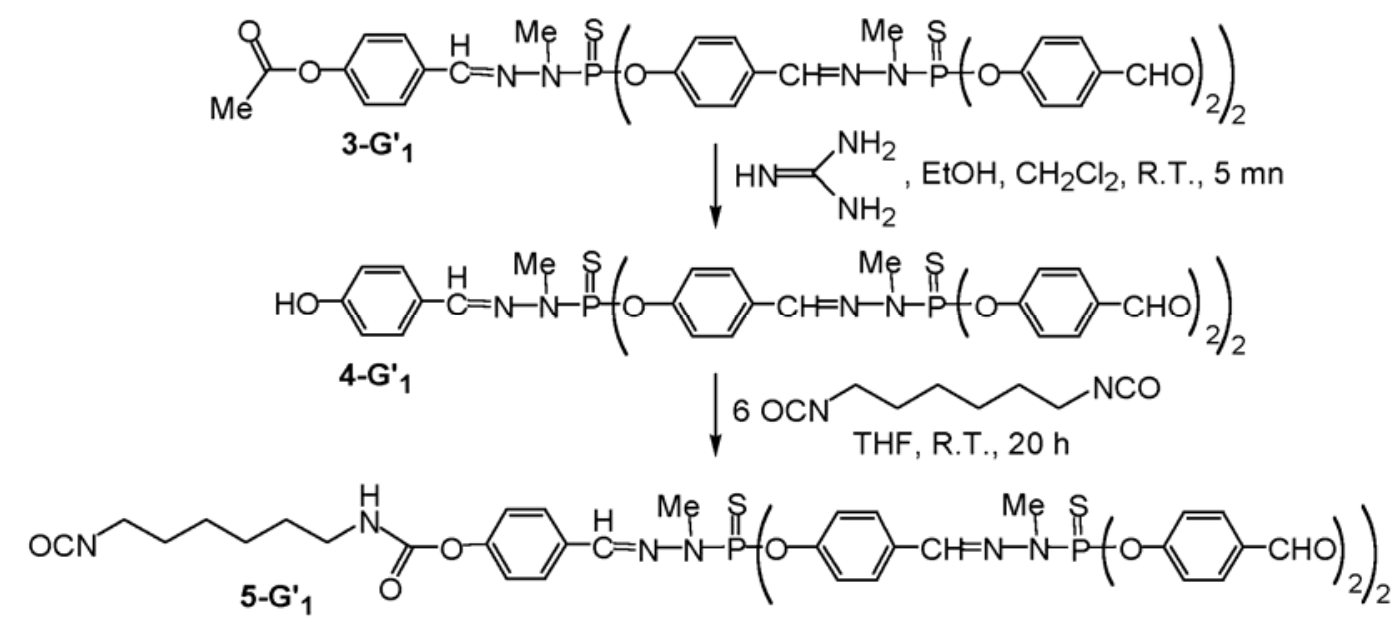

Scheme 3. Reactivity at the core of the dendron $\mathbf{3}-\mathbf{G}^{\prime}{ }_{\mathbf{1}}$.

\section{Conclusions}

We have synthesized two small dendrons and studied the reactivity of the functionality located at

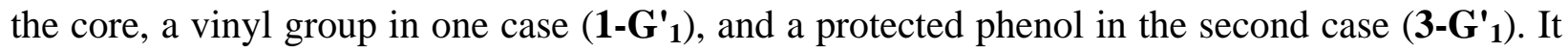
appears that, for practical reasons, Diels-Alder reaction is not the best way to modify the core of dendron 1-G' ${ }_{\mathbf{1}}$, even if the resulting product is isolated in good yield. On the other hand, the very easy deprotection of the phenol group of $\mathbf{3}-\mathbf{G}_{\mathbf{1}}$, leading to $\mathbf{4}-\mathbf{G}_{\mathbf{1}}{ }_{\mathbf{1}}$, should open the way to a versatile reactivity. Indeed, we have shown that dendron $\mathbf{4}-\mathbf{G}_{\mathbf{1}}$ adds easily to isocyanate functionalities, and it is well known that phenols offer a very large palette of reactions. Thus, 
dendron 4-G' 1 should be considered as an $\mathrm{AB}_{4}$ monomer (with $\mathrm{A}=\mathrm{OH}$ and $\mathrm{B}=\mathrm{CHO}$ ) suitable for the synthesis of compounds possessing complex macromolecular dendritic architectures, or for the grafting on various compounds, polymers, or materials.

\section{Experimental Section}

General Procedures. All reactions were carried out in the absence of air, under argon, using standard Schlenk techniques and vacuum-line manipulations. All solvents were dried and distilled before use. Perkin Elmer 1725X was used for FT-IR. NMR spectra were recorded on Bruker AC80, AC200, or AM250 for ${ }^{1} \mathrm{H},{ }^{13} \mathrm{C}$, and ${ }^{31} \mathrm{P}$ NMR, with $\mathrm{SiMe}_{4}$, and $\mathrm{H}_{3} \mathrm{PO}_{4}$ as references, respectively. The attribution of ${ }^{13} \mathrm{C}$ NMR signals was done using Jmod, twodimensional HMBC and HMQC, broad band or $\mathrm{CW}{ }^{31} \mathrm{P}$ decoupling experiments when necessary. The numbering scheme used for NMR is depicted in Figure 2. Compound $\mathbf{1}-\mathbf{G}_{\mathbf{1}}$ was synthesised according to a published procedure. ${ }^{7 \mathrm{a}, 7 \mathrm{~d}}$
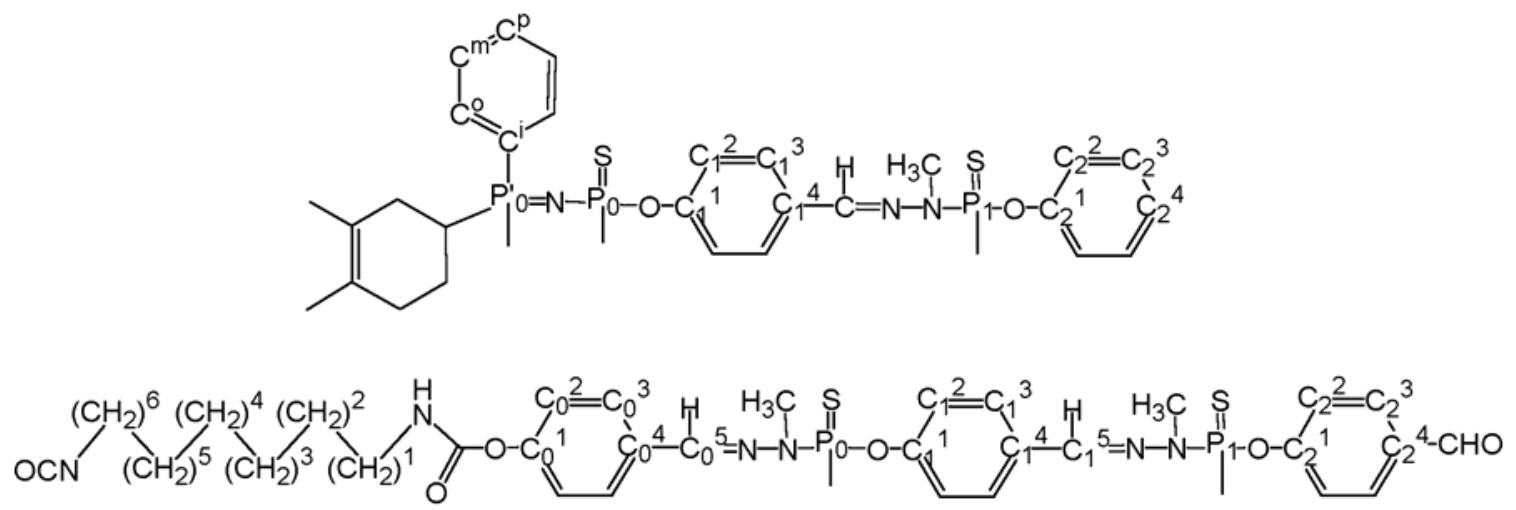

Figure 2. Numbering used for NMR.

Synthesis of dendron 1-G' ${ }_{\mathbf{1}}$. To a solution of dendron $\mathbf{1 - \mathbf { G } _ { \mathbf { 1 } }}(0.2 \mathrm{~g}, 0.234 \mathrm{mmol})$ in THF $(10 \mathrm{~mL})$ was added a slight excess of $\mathrm{NaOPh}(0.11 \mathrm{~g}, 0.95 \mathrm{mmol})$. The resulting mixture was stirred overnight at room temperature, then centrifuged. The solution was recovered and evaporated to dryness to afford a powder, which was washed several times with THF/pentane (1:5) to yield dendron 1-G' 1 .

1-G' ${ }_{1}$ Pale beige powder. $88 \%$ yield. ${ }^{31} \mathrm{P}\left\{{ }^{1} \mathrm{H}\right\}$ NMR $\left(\mathrm{CDCl}_{3}\right): \delta 9.6\left(\mathrm{~d},{ }^{2} \mathrm{~J}_{\mathrm{PP}}=31.3 \mathrm{~Hz}, \mathrm{P}_{0}{ }_{0}\right), 52.2$ $\left(\mathrm{d},{ }^{2} \mathrm{~J}_{\mathrm{PP}}=31.3 \mathrm{~Hz}, \mathrm{P}_{0}\right), 61.8\left(\mathrm{~s}, \mathrm{P}_{\mathrm{l}}\right) ;{ }^{1} \mathrm{H} \mathrm{NMR}\left(\mathrm{CDCl}_{3}\right): \delta 3.3\left(6 \mathrm{H}, \mathrm{d},{ }^{3} \mathrm{~J}_{\mathrm{HP}}=10.6 \mathrm{~Hz}, \mathrm{CH}_{3} \mathrm{~N}\right), 6.1$ $\left(1 \mathrm{H}, \mathrm{ddd},{ }^{3} \mathrm{~J}_{\mathrm{HP}}=24.1 \mathrm{~Hz},{ }^{3} \mathrm{~J}_{\mathrm{HHtrans}}=18.3 \mathrm{~Hz},{ }^{5} \mathrm{~J}_{\mathrm{HP}}=1.1 \mathrm{~Hz}, \mathrm{CH}_{2 \text { trans }}=\mathrm{CH}\right), 6.4\left(1 \mathrm{H}, \mathrm{ddd},{ }^{3} \mathrm{~J}_{\mathrm{HP}}=\right.$ $\left.45.9 \mathrm{~Hz},{ }^{3} \mathrm{~J}_{\mathrm{HHcis}}=12.4 \mathrm{~Hz},{ }^{5} \mathrm{~J}_{\mathrm{HP}}=1.1 \mathrm{~Hz}, \mathrm{CH}_{2 \mathrm{cis}}=\mathrm{CH}\right), 6.8\left(1 \mathrm{H}, \mathrm{dddd},{ }^{2} \mathrm{~J}_{\mathrm{HP}}=25.2 \mathrm{~Hz},{ }^{3} \mathrm{~J}_{\mathrm{HHtrans}}=\right.$ $\left.18.3 \mathrm{~Hz},{ }^{3} \mathrm{~J}_{\mathrm{HHcis}}=12.4 \mathrm{~Hz},{ }^{4} \mathrm{~J}_{\mathrm{HP}}=1.1 \mathrm{~Hz}, \mathrm{CH}=\right), 7.2-7.7\left(40 \mathrm{H}, \mathrm{m}, \mathrm{C}_{6} \mathrm{H}_{5}, \mathrm{C}_{6} \mathrm{H}_{4}, \mathrm{CH}=\mathrm{N}\right) ;{ }^{13} \mathrm{C}\left\{{ }^{1} \mathrm{H}\right\}$ NMR $\left(\mathrm{CDCl}_{3}\right): \delta 32.9\left(\mathrm{~d},{ }^{2} \mathrm{~J}_{\mathrm{CP}}=13.1 \mathrm{~Hz}, \mathrm{P}_{1}-\mathrm{NCH}_{3}\right), 121.3\left(\mathrm{~d},{ }^{3} \mathrm{~J}_{\mathrm{CP}}=4.3 \mathrm{~Hz}, \mathrm{C}_{1}{ }^{2}\right), 121.8\left(\mathrm{~d},{ }^{3} \mathrm{~J}_{\mathrm{CP}}\right.$ $\left.=4.7 \mathrm{~Hz}, \mathrm{C}_{0}{ }^{2}\right), 125.2\left(\mathrm{~s}, \mathrm{C}_{1}^{4}\right), 127.8\left(\mathrm{dd},{ }^{1} \mathrm{~J}_{\mathrm{CP}}=100.0 \mathrm{~Hz},{ }^{3} \mathrm{~J}_{\mathrm{CP}}=4.3 \mathrm{~Hz}, \mathrm{C}^{\mathrm{i}}\right), 127.8\left(\mathrm{~s}, \mathrm{C}_{0}{ }^{3}\right), 128.6$ 
$\left(\mathrm{d},{ }^{2} \mathrm{~J}_{\mathrm{CP}}=13.1 \mathrm{~Hz}, \mathrm{C}^{0}\right), 129.3\left(\mathrm{~s}, \mathrm{C}_{1}{ }^{4}\right), 130.9\left(\mathrm{~s}, \mathrm{C}_{0}^{4}\right), 130.4\left(\mathrm{dd},{ }^{1} \mathrm{~J}_{\mathrm{CP}}=135.9 \mathrm{~Hz},{ }^{3} \mathrm{~J}_{\mathrm{CP}}=4.7 \mathrm{~Hz}\right.$, $\mathrm{CH}=), 132.0\left(\mathrm{~d},{ }^{3} \mathrm{~J}_{\mathrm{CP}}=10.5 \mathrm{~Hz}, \mathrm{C}^{\mathrm{m}}\right), 132.5\left(\mathrm{~d},{ }^{4} \mathrm{~J}_{\mathrm{CP}}=2.4 \mathrm{~Hz}, \mathrm{C}^{\mathrm{p}}\right), 136.6\left(\mathrm{~s}, \mathrm{CH}_{2}=\right), 138.9\left(\mathrm{~d},{ }^{3} \mathrm{~J}_{\mathrm{CP}}\right.$ $=13.4 \mathrm{~Hz}, \mathrm{CH}=\mathrm{N}), 150.5\left(\mathrm{~d},{ }^{2} \mathrm{~J}_{\mathrm{CP}}=7.2 \mathrm{~Hz}, \mathrm{C}_{0}{ }^{\mathrm{l}}\right), 152.8\left(\mathrm{~d},{ }^{2} \mathrm{~J}_{\mathrm{CP}}=9.2 \mathrm{~Hz}, \mathrm{C}_{1}{ }^{\mathrm{l}}\right)$. Anal. Calcd for $\mathrm{C}_{54} \mathrm{H}_{49} \mathrm{~N}_{5} \mathrm{O}_{6} \mathrm{P}_{4} \mathrm{~S}_{3}$ (1084.10): C, 59.82; H, 4.55; N, 6.46. Found: C, 60.03; H, 4.67; N, 6.32.

Synthesis of dendron 2-G' ${ }_{1}$. To a solution of dendron $\mathbf{1 - G}_{\mathbf{1}}(0.2 \mathrm{~g}, 0.18 \mathrm{mmol})$ in toluene (3 mL) was added a large excess of 2,3-dimethylbutadiene ( $3 \mathrm{~mL}, 26.5 \mathrm{mmol}$ ). The resulting solution was heated at $100{ }^{\circ} \mathrm{C}$ for 4 days in a sealed reactor. Then the solution was evaporated to dryness to give a white powder, which was washed twice with pentane.

2-G' 1 White powder. $82 \%$ yield. ${ }^{31} \mathrm{P}\left\{{ }^{1} \mathrm{H}\right\}$ NMR (THF $d_{8}$ ): $\delta 19.9\left(\mathrm{~d},{ }^{2} \mathrm{~J}_{\mathrm{PP}}=33.2 \mathrm{~Hz}, \mathrm{P}^{\prime}{ }_{0}\right.$ ), 50.7 $\left(\mathrm{d},{ }^{2} \mathrm{~J}_{\mathrm{PP}}=33.2 \mathrm{~Hz}, \mathrm{P}_{0}\right), 61.5\left(\mathrm{~s}, \mathrm{P}_{\mathrm{l}}\right) ;{ }^{1} \mathrm{H}$ NMR (THF $\left.d_{8}\right): \delta 1.64\left(3 \mathrm{H}, \mathrm{s}, \mathrm{CH}_{3}\right), 1.69\left(3 \mathrm{H}, \mathrm{s}, \mathrm{CH}_{3}\right)$, 1.7-2.4 (7H, m, $\left.\mathrm{CH}_{2}, \mathrm{CH}\right), 3.45\left(6 \mathrm{H}, \mathrm{d},{ }^{3} \mathrm{~J}_{\mathrm{HP}}=10.6 \mathrm{~Hz}, \mathrm{CH}_{3} \mathrm{~N}\right), 7.0-8.0\left(40 \mathrm{H}, \mathrm{m}, \mathrm{C}_{6} \mathrm{H}_{5}, \mathrm{C}_{6} \mathrm{H}_{4}\right.$, $\mathrm{CH}=\mathrm{N}) ;{ }^{13} \mathrm{C}\left\{{ }^{1} \mathrm{H}\right\}$ NMR (THF $\left.d_{8}\right): \delta 19.3$ and $19.4\left(\mathrm{~s}, \underline{\mathrm{CH}_{3}}-\mathrm{C}=\right), 23.8\left(\mathrm{~s},=\mathrm{C}-\underline{\mathrm{CH}}_{2}-\mathrm{CH}_{2}\right), 31.1$ (s, $\mathrm{CH}_{2}-\underline{\mathrm{CH}}_{2}-\underline{C H P}_{0}^{\prime}$ ), 32.3 (d, $\left.{ }^{2} \mathrm{~J}_{\mathrm{CP}}=13.8 \mathrm{~Hz},=\mathrm{C}-\underline{\mathrm{CH}}_{2}-\mathrm{CHP}_{0}^{\prime}\right), 33.5\left(\mathrm{~d},{ }^{2} \mathrm{~J}_{\mathrm{CP}}=12.2 \mathrm{~Hz}, \mathrm{P}_{1}-\mathrm{NCH}_{3}\right)$, $34.2\left(\mathrm{~d},{ }^{1} \mathrm{~J}_{\mathrm{CP}}=67.0 \mathrm{~Hz}, \mathrm{CH}-\mathrm{P}\right), 122.5\left(\mathrm{~d},{ }^{3} \mathrm{~J}_{\mathrm{CP}}=4.6 \mathrm{~Hz}, \mathrm{C}_{1}{ }^{2}\right), 123.0\left(\mathrm{~d},{ }^{3} \mathrm{~J}_{\mathrm{CP}}=4 \mathrm{~Hz}, \mathrm{C}_{0}{ }^{2}\right), 125.1$ $\left(\mathrm{d},{ }^{3} \mathrm{~J}_{\mathrm{CP}}=13.8 \mathrm{~Hz},=\underline{\mathrm{C}}-\mathrm{CH}_{2}-\mathrm{CHP}_{0}^{\prime}\right), 126.1\left(\mathrm{~s}, \mathrm{C}_{1}{ }^{4}\right), 128.7\left(\mathrm{~s}, \mathrm{C}_{0}^{3}\right), 129.2\left(\mathrm{dd},{ }^{1} \mathrm{~J}_{\mathrm{CP}}=120 \mathrm{~Hz},{ }^{3} \mathrm{~J}_{\mathrm{CP}}=\right.$ $5.8 \mathrm{~Hz}, \mathrm{C}^{\mathrm{i}}$ ), 129.3 (s, =CH- $\left.\underline{\mathrm{CH}} \underline{2}_{2}-\mathrm{CH}_{2}\right), 129.6\left(\mathrm{~d},{ }^{2} \mathrm{~J}_{\mathrm{CP}}=12 \mathrm{~Hz}, \mathrm{C}^{0}\right), 130.4\left(\mathrm{~s}, \mathrm{C}_{1}^{3}\right), 132.4\left(\mathrm{~s}, \mathrm{C}_{0}^{4}\right)$, $133.3\left(\mathrm{~d},{ }^{3} \mathrm{~J}_{\mathrm{CP}}=10.5 \mathrm{~Hz}, \mathrm{C}^{\mathrm{m}}\right), 133.5\left(\mathrm{~s}, \mathrm{C}^{\mathrm{p}}\right), 140.8\left(\mathrm{~d},{ }^{3} \mathrm{~J}_{\mathrm{CP}}=13.3 \mathrm{~Hz}, \mathrm{CH}=\mathrm{N}\right), 152.2\left(\mathrm{~d},{ }^{2} \mathrm{~J}_{\mathrm{CP}}=7.9\right.$ $\mathrm{Hz}, \mathrm{C}_{0}{ }^{1}$ ), 154.7 (d, ${ }^{2} \mathrm{~J}_{\mathrm{CP}}=7.9 \mathrm{~Hz}, \mathrm{C}_{1}{ }^{1}$ ). Anal. Calcd for $\mathrm{C}_{60} \mathrm{H}_{59} \mathrm{~N}_{5} \mathrm{O}_{6} \mathrm{P}_{4} \mathrm{~S}_{3}$ (1166.24): C, 61.79; H, 5.09; N, 6.00. Found: C, 61.50; H, 4.97; N, 5.81.

\section{General procedure for the synthesis of dendrons $3-G_{n}\left(P(S) C_{2}\right.$ end groups, $\left.n=0,1\right)$}

To a solution of $1 \mathrm{~g}$ of dendron $3-\mathbf{G}_{0}^{\prime}$ (aldehyde end groups) or $\mathrm{MeCO}_{2} \mathrm{C}_{6} \mathrm{H}_{4} \mathrm{CHO} 3$ in chloroform $(20 \mathrm{~mL})$ was added at room temperature $5 \%$ molar excess of a solution of $\mathrm{H}_{2} \mathrm{NNMeP}(\mathrm{S}) \mathrm{Cl}_{2}$ in chloroform $(0.3 \mathrm{M})$. The resulting solution was stirred overnight, then evaporated to dryness to afford $\mathbf{3}-\mathbf{G}_{\mathbf{n}}$ as a white powder, which was washed three times with ether/pentane $1 / 1$.

\section{General procedure for the synthesis of dendrons $3-G^{\prime}{ }_{n}($ aldehyde end groups, $n=0,1)$}

To a solution of $1 \mathrm{~g}$ of dendron $\mathbf{3}-\mathbf{G}_{\mathbf{n}}\left(\mathrm{P}(\mathrm{S}) \mathrm{Cl}_{2}\right.$ end groups) in THF (20 mL) was added at room temperature $3 \%$ molar excess of 4-hydroxybenzaldehyde, sodium salt (powder). The resulting heterogeneous mixture was stirred overnight, then centrifuged (10000 turns per min). The

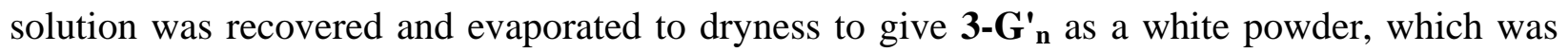
washed three times with ether.

3-G. White powder, 97\% yield. ${ }^{31} \mathrm{P}\left\{{ }^{1} \mathrm{H}\right\} \mathrm{NMR}\left(\mathrm{CDCl}_{3}\right) \delta 63.5 ;{ }^{1} \mathrm{H}$ NMR $\left(\mathrm{CDCl}_{3}\right) \delta 2.31(3 \mathrm{H}$, s, $\left.\mathrm{CH}_{3} \mathrm{CO}\right), 3.48\left(3 \mathrm{H}, \mathrm{d},{ }^{3} \mathrm{~J}_{\mathrm{HP}}=13.9 \mathrm{~Hz}, \mathrm{CH}_{3} \mathrm{NP}_{0}\right), 7.14\left(2 \mathrm{H}, \mathrm{d},{ }^{3} \mathrm{~J}_{\mathrm{HH}}=8.6 \mathrm{~Hz}, \mathrm{HC}_{0}{ }^{2}\right), 7.64(1 \mathrm{H}, \mathrm{d}$, $\left.{ }^{4} \mathrm{~J}_{\mathrm{HP}}=2.6 \mathrm{~Hz}, \mathrm{HC}_{0}{ }^{5}\right), 7.75\left(2 \mathrm{H}, \mathrm{d},{ }^{3} \mathrm{~J}_{\mathrm{HH}}=8.6 \mathrm{~Hz}, \mathrm{HC}_{0}{ }^{3}\right) ;{ }^{13} \mathrm{C}\left\{{ }^{1} \mathrm{H}\right\} \mathrm{NMR}\left(\mathrm{CDCl}_{3}\right) \delta 21.2(\mathrm{~s}$, $\left.\mathrm{CH}_{3} \mathrm{CO}\right), 31.8\left(\mathrm{~d},{ }^{2} \mathrm{~J}_{\mathrm{CP}}=13.5 \mathrm{~Hz}, \mathrm{CH}_{3} \mathrm{NP}_{0}\right), 122.1\left(\mathrm{~s}, \mathrm{C}_{0}{ }^{2}\right), 128.5\left(\mathrm{~s}, \mathrm{C}_{0}{ }^{3}\right), 131.8\left(\mathrm{~s}, \mathrm{C}_{0}{ }^{4}\right), 140.8(\mathrm{~d}$, ${ }^{3} \mathrm{~J}_{\mathrm{CP}}=17.9 \mathrm{~Hz}, \mathrm{C}_{0}^{5}$ ), 152.0 (s, $\mathrm{C}_{0}^{1}$ ), 169.2 (s, $\mathrm{CH}_{3} \mathrm{CO}$ ). Anal. Calcd for $\mathrm{C}_{10} \mathrm{H}_{11} \mathrm{~N}_{2} \mathrm{Cl}_{2} \mathrm{O}_{2} \mathrm{PS}$ (325.1): C, 36.94; H, 3.41; N, 8.61. Found: C, 36.39; H, 2.99; N, 8.21. 
3-G' ${ }_{0}$. White powder, 98\% yield. ${ }^{31} \mathrm{P}\left\{{ }^{1} \mathrm{H}\right\}$ NMR $\left(\mathrm{CDCl}_{3}\right) \delta 60.6 ;{ }^{1} \mathrm{H}$ NMR $\left(\mathrm{CDCl}_{3}\right) \delta 2.30(3 \mathrm{H}$, s, $\left.\mathrm{CH}_{3} \mathrm{CO}\right), 3.37\left(3 \mathrm{H}, \mathrm{d},{ }^{3} \mathrm{~J}_{\mathrm{HP}}=11.0 \mathrm{~Hz}, \mathrm{CH}_{3} \mathrm{NP}_{0}\right), 7.10\left(2 \mathrm{H}, \mathrm{d},{ }^{3} \mathrm{~J}_{\mathrm{HH}}=8.6 \mathrm{~Hz}, \mathrm{HC}_{0}{ }^{2}\right), 7.36(4 \mathrm{H}$, $\left.\mathrm{dd},{ }^{4} \mathrm{~J}_{\mathrm{HP}}=1.5 \mathrm{~Hz},{ }^{3} \mathrm{~J}_{\mathrm{HH}}=8.5 \mathrm{~Hz}, \mathrm{HC}_{1}{ }^{2}\right), 7.65\left(1 \mathrm{H}, \mathrm{s}, \mathrm{HC}_{0}^{5}\right), 7.67\left(2 \mathrm{H}, \mathrm{d},{ }^{3} \mathrm{~J}_{\mathrm{HH}}=8.6 \mathrm{~Hz}, \mathrm{HC}_{0}{ }^{3}\right)$, $7.86\left(4 \mathrm{H}, \mathrm{d},{ }^{3} \mathrm{~J}_{\mathrm{HH}}=8.5 \mathrm{~Hz}, \mathrm{HC}_{1}{ }^{3}\right), 9.94(2 \mathrm{H}, \mathrm{s}, \mathrm{CHO}) ;{ }^{13} \mathrm{C}\left\{{ }^{1} \mathrm{H}\right\} \mathrm{NMR}\left(\mathrm{CDCl}_{3}\right) \delta 21.2(\mathrm{~s}$, $\mathrm{CH}_{3} \mathrm{CO}$ ), $32.9\left(\mathrm{~d},{ }^{2} \mathrm{~J}_{\mathrm{CP}}=13.8 \mathrm{~Hz}, \mathrm{CH}_{3} \mathrm{NP}_{0}\right), 122.0\left(\mathrm{~s}, \mathrm{C}_{0}{ }^{2}\right), 122.1\left(\mathrm{~d},{ }^{3} \mathrm{~J}_{\mathrm{CP}}=3.5 \mathrm{~Hz}, \mathrm{C}_{1}{ }^{2}\right), 128.1(\mathrm{~s}$, $\left.\mathrm{C}_{0}^{3}, \mathrm{C}_{1}^{3}\right), 131.5\left(\mathrm{~s}, \mathrm{C}_{0}{ }^{4}, \mathrm{C}_{1}^{4}\right), 139.8\left(\mathrm{~d},{ }^{3} \mathrm{~J}_{\mathrm{CP}}=13.8 \mathrm{~Hz}, \mathrm{C}_{0}{ }^{5}\right), 151.7\left(\mathrm{~s}, \mathrm{C}_{0}{ }^{1}\right), 155.1\left(\mathrm{~d},{ }^{3} \mathrm{~J}_{\mathrm{CP}}=8.2\right.$ $\mathrm{Hz}, \mathrm{C}_{1}^{1}$ ), 169.2 (s, $\mathrm{CH}_{3} \underline{\mathrm{CO}}$ ), 190.9 (s, CHO). Anal. Calcd for $\mathrm{C}_{24} \mathrm{H}_{21} \mathrm{~N}_{2} \mathrm{O}_{6} \mathrm{PS}$ (496.5): C, 58.06; H, 4.26; N, 5.64. Found: C, 57.80; H, 3.98; N, 5.33.

3-G . White powder, 97\% yield. ${ }^{31} \mathrm{P}\left\{{ }^{1} \mathrm{H}\right\}$ NMR $\left(\mathrm{CDCl}_{3}\right) \delta 61.4\left(\mathrm{~s}, \mathrm{P}_{0}\right), 62.5\left(\mathrm{~s}, \mathrm{P}_{1}\right) ;{ }^{1} \mathrm{H}$ NMR $\left(\mathrm{CDCl}_{3}\right) \delta 2.30\left(3 \mathrm{H}, \mathrm{s}, \mathrm{CH}_{3} \mathrm{CO}\right), 3.36\left(3 \mathrm{H}, \mathrm{d},{ }^{3} \mathrm{~J}_{\mathrm{HP}}=10.8 \mathrm{~Hz}, \mathrm{CH}_{3} \mathrm{NP}_{0}\right), 3.47\left(6 \mathrm{H}, \mathrm{d},{ }^{3} \mathrm{~J}_{\mathrm{HP}}=13.9\right.$ $\left.\mathrm{Hz}, \mathrm{CH}_{3} \mathrm{NP}_{1}\right), 7.14\left(2 \mathrm{H}, \mathrm{d},{ }^{3} \mathrm{~J}_{\mathrm{HH}}=8.6 \mathrm{~Hz}, \mathrm{HC}_{0}^{2}\right), 7.28\left(4 \mathrm{H}, \mathrm{m}, \mathrm{HC}_{1}^{2}\right), 7.60-7.77\left(7 \mathrm{H}, \mathrm{m}, \mathrm{HC}_{0}^{5}\right.$, $\left.\mathrm{HC}_{0}{ }^{3}, \mathrm{HC}_{1}{ }^{3}\right) ;{ }^{13} \mathrm{C}\left\{{ }^{1} \mathrm{H}\right\}$ NMR $\left(\mathrm{CDCl}_{3}\right) \delta 21.2\left(\mathrm{~s}, \underline{\mathrm{CH}}_{3} \mathrm{CO}\right), 31.8\left(\mathrm{~d},{ }^{2} \mathrm{~J}_{\mathrm{CP}}=13.3 \mathrm{~Hz}, \mathrm{CH}_{3} \mathrm{NP}_{1}\right), 33.1$ $\left(\mathrm{d},{ }^{2} \mathrm{~J}_{\mathrm{CP}}=13.3 \mathrm{~Hz}, \mathrm{CH}_{3} \mathrm{NP}_{0}\right), 122.0\left(\mathrm{~d},{ }^{3} \mathrm{~J}_{\mathrm{CP}}=4.5 \mathrm{~Hz}, \mathrm{C}_{1}{ }^{2}\right), 122.1\left(\mathrm{~s}, \mathrm{C}_{0}{ }^{2}\right), 128.1\left(\mathrm{~s}, \mathrm{C}_{0}{ }^{3}, \mathrm{C}_{1}{ }^{3}\right)$, $131.5\left(\mathrm{~s}, \mathrm{C}_{0}{ }^{4}, \mathrm{C}_{1}^{4}\right), 139.1\left(\mathrm{~d},{ }^{3} \mathrm{~J}_{\mathrm{CP}}=16.7 \mathrm{~Hz}, \mathrm{C}_{0}{ }^{5}\right), 140.8\left(\mathrm{~d},{ }^{3} \mathrm{~J}_{\mathrm{CP}}=18.4 \mathrm{~Hz}, \mathrm{C}_{1}{ }^{5}\right), 151.7\left(\mathrm{~s}, \mathrm{C}_{0}{ }^{1}\right)$, $155.1\left(\mathrm{~d},{ }^{3} \mathrm{~J}_{\mathrm{CP}}=8.2 \mathrm{~Hz}, \mathrm{C}_{1}{ }^{1}\right.$ ), 169.3 (s, $\mathrm{CH}_{3} \mathrm{CO}$ ). Anal. Calcd for $\mathrm{C}_{26} \mathrm{H}_{27} \mathrm{~N}_{6} \mathrm{Cl}_{4} \mathrm{O}_{4} \mathrm{P}_{3} \mathrm{~S}_{3}$ (818.5): C, 38.15; H, 3.32; N, 10.27. Found: C, 37.89; H, 3.07; N, 9.97.

3-G' ${ }_{1}$. White powder, 98\% yield. ${ }^{31} \mathrm{P}\left\{{ }^{1} \mathrm{H}\right\}$ NMR $\left(\mathrm{CDCl}_{3}\right) \delta 60.8\left(\mathrm{~s}, \mathrm{P}_{1}\right), 62.7\left(\mathrm{~s}, \mathrm{P}_{0}\right) ;{ }^{1} \mathrm{H}$ NMR $\left(\mathrm{CDCl}_{3}\right) \delta 2.30$ (3H, s, $\left.\mathrm{CH}_{3} \mathrm{CO}\right), 3.37\left(3 \mathrm{H}, \mathrm{d},{ }^{3} \mathrm{~J}_{\mathrm{HP}}=10.7 \mathrm{~Hz}, \mathrm{CH}_{3} \mathrm{NP}_{0}\right), 3.38\left(6 \mathrm{H}, \mathrm{d},{ }^{3} \mathrm{~J}_{\mathrm{HP}}=10.7\right.$ $\left.\mathrm{Hz}, \mathrm{CH}_{3} \mathrm{NP}_{1}\right), 7.12\left(2 \mathrm{H}, \mathrm{d},{ }^{3} \mathrm{~J}_{\mathrm{HH}}=8.6 \mathrm{~Hz}, \mathrm{HC}_{0}{ }^{2}\right), 7.25\left(4 \mathrm{H}, \mathrm{dd},{ }^{4} \mathrm{~J}_{\mathrm{HP}}=1.6 \mathrm{~Hz},{ }^{3} \mathrm{~J}_{\mathrm{HH}}=8.6 \mathrm{~Hz}\right.$, $\left.\mathrm{HC}_{1}^{2}\right) ; 7.36\left(4 \mathrm{H}, \mathrm{dd},{ }^{4} \mathrm{~J}_{\mathrm{HP}}=1.3 \mathrm{~Hz},{ }^{3} \mathrm{~J}_{\mathrm{HH}}=8.4 \mathrm{~Hz}, \mathrm{HC}_{2}{ }^{2}\right), 7.60-7.70\left(7 \mathrm{H}, \mathrm{m}, \mathrm{HC}_{1}{ }^{3}, \mathrm{HC}_{0}{ }^{5}, \mathrm{HC}_{1}{ }^{5}\right)$, $7.74\left(2 \mathrm{H}, \mathrm{d},{ }^{3} \mathrm{~J}_{\mathrm{HH}}=8.6 \mathrm{~Hz}, \mathrm{HC}_{0}{ }^{3}\right), 7.85\left(8 \mathrm{H}, \mathrm{d},{ }^{3} \mathrm{~J}_{\mathrm{HH}}=8.4 \mathrm{~Hz}, \mathrm{HC}_{2}{ }^{3}\right), 9.93(4 \mathrm{H}, \mathrm{s}, \mathrm{CHO}) ;{ }^{13} \mathrm{C}$ $\left\{{ }^{1} \mathrm{H}\right\}$ NMR $\left(\mathrm{CDCl}_{3}\right) \delta 21.1\left(\mathrm{~s}, \underline{\mathrm{CH}}_{3} \mathrm{CO}\right), 32.9\left(\mathrm{~d},{ }^{2} \mathrm{~J}_{\mathrm{CP}}=13.1 \mathrm{~Hz}, \mathrm{CH}_{3} \mathrm{NP}_{1}\right), 33.1\left(\mathrm{~d},{ }^{2} \mathrm{~J}_{\mathrm{CP}}=9.9 \mathrm{~Hz}\right.$, $\mathrm{CH}_{3} \mathrm{NP}_{0}$ ); 122.0 (m, $\left.\mathrm{C}_{0}^{2}, \mathrm{C}_{1}{ }^{2}, \mathrm{C}_{2}{ }^{2}\right), 128.1\left(\mathrm{~s}, \mathrm{C}_{0}^{3}\right), 128.3\left(\mathrm{~s}, \mathrm{C}_{1}^{3}\right), 131.5\left(\mathrm{~s}, \mathrm{C}_{2}^{3}\right), 132.2\left(\mathrm{~s}, \mathrm{C}_{0}^{4}\right)$, $132.4\left(\mathrm{~s}, \mathrm{C}_{1}^{4}\right), 133.6\left(\mathrm{~s}, \mathrm{C}_{2}{ }^{4}\right), 139.1\left(\mathrm{~d},{ }^{3} \mathrm{~J}_{\mathrm{CP}}=13.7 \mathrm{~Hz}, \mathrm{C}_{0}{ }^{5}\right), 139.6\left(\mathrm{~d},{ }^{3} \mathrm{~J}_{\mathrm{CP}}=13.7 \mathrm{~Hz}, \mathrm{C}_{1}{ }^{5}\right), 151.5$ (m, $\left.\mathrm{C}_{0}{ }^{1}, \mathrm{C}_{1}{ }^{1}\right), 155.2\left(\mathrm{~d},{ }^{3} \mathrm{~J}_{\mathrm{CP}}=6.7 \mathrm{~Hz}, \mathrm{C}_{2}{ }^{1}\right), 169.4\left(\mathrm{~s}, \mathrm{CH}_{3} \underline{\mathrm{CO}}\right), 190.8$ (s, CHO) ppm. Anal. Calcd for $\mathrm{C}_{54} \mathrm{H}_{47} \mathrm{~N}_{6} \mathrm{O}_{12} \mathrm{P}_{3} \mathrm{~S}_{3}$ (1161.1): C, 55.86; H, 4.08; N, 7.24. Found: C, 55.49; H, 3.87; N, 7.03.

Synthesis of dendron 4-G' ${ }_{1}$. A freshly prepared solution of guanidine, obtained from guanidine hydrochloride ( $0.026 \mathrm{~g}, 0.44 \mathrm{mmol})$ and sodium ethoxide $(0.03 \mathrm{~g}, 0.44 \mathrm{mmol})$ in ethanol $(10 \mathrm{~mL})$ was added to a solution of dendron 3-G' $10.50 \mathrm{~g}, 0.43 \mathrm{mmol})$ in $\mathrm{CH}_{2} \mathrm{Cl}_{2}(10 \mathrm{~mL})$ ). The resulting mixture was stirred for 5 minutes, the solvents were removed under vacuum. The resulting powder was dissolved in $\mathrm{CH}_{2} \mathrm{Cl}_{2}(10 \mathrm{~mL})$, then dendron $\mathbf{4}-\mathbf{G}_{\mathbf{1}}$ was purified by flash chromatography.

4-G' ${ }_{1}$. White powder, 89\% yield. ${ }^{31} \mathrm{P}\left\{{ }^{1} \mathrm{H}\right\}$ NMR $\left(\mathrm{CDCl}_{3}\right) \delta 60.1\left(\mathrm{~s}, \mathrm{P}_{1}\right), 61.9\left(\mathrm{~s}, \mathrm{P}_{0}\right) ;{ }^{1} \mathrm{H}$ NMR $\left(\mathrm{CDCl}_{3}\right) \delta 3.33\left(3 \mathrm{H}, \mathrm{d},{ }^{3} \mathrm{~J}_{\mathrm{HP}}=10.6 \mathrm{~Hz}, \mathrm{CH}_{3} \mathrm{NP}_{0}\right), 3,37\left(6 \mathrm{H}, \mathrm{d},{ }^{3} \mathrm{~J}_{\mathrm{HP}}=10.9 \mathrm{~Hz}, \mathrm{CH}_{3} \mathrm{NP}_{1}\right), 6.85$ $\left(2 \mathrm{H}, \mathrm{d},{ }^{3} \mathrm{~J}_{\mathrm{HH}}=8.5 \mathrm{~Hz}, \mathrm{HC}_{0}^{2}\right), 7.25\left(4 \mathrm{H}, \mathrm{dd},{ }^{4} \mathrm{~J}_{\mathrm{HP}}=1.4 \mathrm{~Hz},{ }^{3} \mathrm{~J}_{\mathrm{HH}}=8.3 \mathrm{~Hz}, \mathrm{HC}_{1}{ }^{2}\right), 7.35\left(8 \mathrm{H}, \mathrm{d},{ }^{3} \mathrm{~J}_{\mathrm{HH}}\right.$ $\left.=8.4 \mathrm{~Hz}, \mathrm{HC}_{2}^{2}\right), 7.50-7.70\left(9 \mathrm{H}, \mathrm{m}, \mathrm{HC}_{0}^{3}, \mathrm{HC}_{1}^{3}, \mathrm{HC}_{0}{ }^{5}, \mathrm{HC}_{1}^{5}\right), 7.85\left(8 \mathrm{H}, \mathrm{d},{ }^{3} \mathrm{~J}_{\mathrm{HH}}=8.4 \mathrm{~Hz}, \mathrm{HC}_{2}^{3}\right)$, $9.92(4 \mathrm{H}, \mathrm{s}, \mathrm{CHO}) ;{ }^{13} \mathrm{C}\left\{{ }^{1} \mathrm{H}\right\} \mathrm{NMR}\left(\mathrm{CDCl}_{3}\right) \delta 21.1\left(\mathrm{~s}, \underline{\mathrm{CH}}_{3} \mathrm{CO}\right), 32.9\left(\mathrm{~d},{ }^{2} \mathrm{~J}_{\mathrm{CP}}=13.1 \mathrm{~Hz}\right.$, $\left.\mathrm{CH}_{3} \mathrm{NP}_{1}\right), 33.1\left(\mathrm{~d},{ }^{2} \mathrm{~J}_{\mathrm{CP}}=9.9 \mathrm{~Hz}, \mathrm{CH}_{3} \mathrm{NP}_{0}\right), 122.0\left(\mathrm{~m}, \mathrm{C}_{0}{ }^{2}, \mathrm{C}_{1}{ }^{2}, \mathrm{C}_{2}{ }^{2}\right), 128.1\left(\mathrm{~s}, \mathrm{C}_{0}{ }^{3}\right), 128.3\left(\mathrm{~s}, \mathrm{C}_{1}{ }^{3}\right)$, $131.5\left(\mathrm{~s}, \mathrm{C}_{2}{ }^{3}\right), 132.2\left(\mathrm{~s}, \mathrm{C}_{0}^{4}\right), 132.4\left(\mathrm{~s}, \mathrm{C}_{1}{ }^{4}\right), 133.6\left(\mathrm{~s}, \mathrm{C}_{2}{ }^{4}\right), 139.1\left(\mathrm{~d},{ }^{3} \mathrm{~J}_{\mathrm{CP}}=13.7 \mathrm{~Hz}, \mathrm{C}_{0}{ }^{5}\right), 139.6$ $\left(\mathrm{d},{ }^{3} \mathrm{~J}_{\mathrm{CP}}=13.7 \mathrm{~Hz}, \mathrm{C}_{1}^{5}\right), 151.5\left(\mathrm{~m}, \mathrm{C}_{0}{ }^{1}, \mathrm{C}_{1}{ }^{1}\right), 155.2\left(\mathrm{~d},{ }^{3} \mathrm{~J}_{\mathrm{CP}}=6.7 \mathrm{~Hz}, \mathrm{C}_{2}{ }^{1}\right), 190.8$ (s, CHO). MS 
(FAB) m/z: $1119[\mathrm{MH}]^{+}$. Anal. Calcd for $\mathrm{C}_{52} \mathrm{H}_{45} \mathrm{~N}_{6} \mathrm{O}_{11} \mathrm{P}_{3} \mathrm{~S}_{3}$ (1119.1): C, 55.81; H, 4.05; N, 7.51. Found: C, 55.49; H, 3.89; N, 7.25.

Synthesis of dendron 5-G' ${ }_{1}$. To a solution of dendron $\left.4-\mathbf{G}_{\mathbf{1}} \mathbf{1}_{\text {(0.15 }} \mathbf{g}, 0.134 \mathrm{mmol}\right)$ in THF (10 $\mathrm{mL}$ ) were added 1 equivalent of triethylamine $(20 \mu \mathrm{L}, 0.15 \mathrm{mmol})$ and 6 équivalents of diisocyanohexane $(130 \mu \mathrm{L}, 0.80 \mathrm{mmol})$. The resulting solution was stirred overnight at room temperature, then evaporated to dryness, to afford a powder, which was washed with $\mathrm{Et}_{2} \mathrm{O}$ till the obtaining of $\mathbf{5}-\mathbf{G}_{\mathbf{1}}$ as a white powder.

5-G' ${ }_{1}$. White powder, $89 \%$ yield. ${ }^{31} \mathrm{P}\left\{{ }^{1} \mathrm{H}\right\} \mathrm{NMR}\left(\mathrm{CDCl}_{3}\right) \delta 60.1\left(\mathrm{~s}, \mathrm{P}_{1}\right), 61.9\left(\mathrm{~s}, \mathrm{P}_{0}\right) ;{ }^{1} \mathrm{H}$ NMR $\left(\mathrm{CDCl}_{3}\right) \delta 1.38\left(4 \mathrm{H}, \mathrm{m},\left(\mathrm{CH}_{2}\right)^{3},\left(\mathrm{CH}_{2}\right)^{4}\right), 1.59\left(4 \mathrm{H}, \mathrm{m},\left(\mathrm{CH}_{2}\right)^{2},\left(\mathrm{CH}_{2}\right)^{5}\right), 3.20\left(4 \mathrm{H}, \mathrm{m},\left(\mathrm{CH}_{2}\right)^{1}\right.$, $\left.\left(\mathrm{CH}_{2}\right)^{6}\right), 3.32\left(3 \mathrm{H}, \mathrm{d},{ }^{3} \mathrm{~J}_{\mathrm{HP}}=10.6 \mathrm{~Hz}, \mathrm{CH}_{3} \mathrm{NP}_{0}\right), 3.37\left(6 \mathrm{H}, \mathrm{d},{ }^{3} \mathrm{~J}_{\mathrm{HP}}=10.9 \mathrm{~Hz}, \mathrm{CH}_{3} \mathrm{NP}_{1}\right), 5.42(1 \mathrm{H}$, $\left.\mathrm{t},{ }^{3} \mathrm{~J}_{\mathrm{HH}}=6.0 \mathrm{~Hz}, \mathrm{HNCO}\right), 7.15\left(2 \mathrm{H}, \mathrm{d},{ }^{3} \mathrm{~J}_{\mathrm{HH}}=8.5 \mathrm{~Hz}, \mathrm{HC}_{0}{ }^{2}\right), 7.25\left(4 \mathrm{H}, \mathrm{dd},{ }^{4} \mathrm{~J}_{\mathrm{HP}}=1.2 \mathrm{~Hz},{ }^{3} \mathrm{~J}_{\mathrm{HH}}=\right.$ $\left.8.5 \mathrm{~Hz}, \mathrm{HC}_{1}^{2}\right), 7.36\left(8 \mathrm{H}, \mathrm{dd},{ }^{4} \mathrm{~J}_{\mathrm{HP}}=1.0 \mathrm{~Hz},{ }^{3} \mathrm{~J}_{\mathrm{HH}}=8.5 \mathrm{~Hz}, \mathrm{HC}_{2}{ }^{2}\right), 7.60-7.70\left(9 \mathrm{H}, \mathrm{m}, \mathrm{HC}_{0}{ }^{3}, \mathrm{HC}_{1}{ }^{3}\right.$, $\left.\mathrm{HC}_{0}{ }^{5}, \mathrm{HC}_{1}{ }^{5}\right), 7.85\left(8 \mathrm{H}, \mathrm{d},{ }^{3} \mathrm{~J}_{\mathrm{HH}}=8.5 \mathrm{~Hz}, \mathrm{HC}_{2}{ }^{3}\right), 9.93(4 \mathrm{H}, \mathrm{s}, \mathrm{CHO}) ;{ }^{13} \mathrm{C}\left\{{ }^{1} \mathrm{H}\right\} \mathrm{NMR}\left(\mathrm{CDCl}_{3}\right) \delta$ 25.9 and $26.1\left(2 \mathrm{~s},\left(\mathrm{CH}_{2}\right)^{3}\right.$ and $\left.\left(\mathrm{CH}_{2}\right)^{4}\right), 29.6\left(\mathrm{~s},\left(\mathrm{CH}_{2}\right)^{2}\right), 31.0\left(\mathrm{~s},\left(\mathrm{CH}_{2}\right)^{5}\right), 32.9\left(\mathrm{~d},{ }^{2} \mathrm{~J}_{\mathrm{CP}}=13.1 \mathrm{~Hz}\right.$, $\left.\mathrm{CH}_{3} \mathrm{NP}_{1}\right), 33.1\left(\mathrm{~d},{ }^{2} \mathrm{~J}_{\mathrm{CP}}=9.9 \mathrm{~Hz}, \mathrm{CH}_{3} \mathrm{NP}_{0}\right), 41,1\left(\mathrm{~s},\left(\mathrm{CH}_{2}\right)^{1}\right), 42.8\left(\mathrm{~s},\left(\mathrm{CH}_{2}\right)^{6}\right), 121.9\left(\mathrm{~m}, \mathrm{C}_{0}{ }^{2}, \mathrm{C}_{1}{ }^{2}\right.$, $\mathrm{C}_{2}{ }^{2}$, NCO), $128.0\left(\mathrm{~s}, \mathrm{C}_{0}{ }^{3}\right), 128.3\left(\mathrm{~s}, \mathrm{C}_{1}{ }^{3}\right), 131.5\left(\mathrm{~s}, \mathrm{C}_{2}{ }^{3}\right), 131.7\left(\mathrm{~s}, \mathrm{C}_{0}{ }^{4}, \mathrm{C}_{1}{ }^{4}\right), 133.6\left(\mathrm{~s}_{2} \mathrm{C}_{2}{ }^{4}\right), 139.3$ $\left(\mathrm{d},{ }^{3} \mathrm{~J}_{\mathrm{CP}}=13.7 \mathrm{~Hz}, \mathrm{C}_{0}^{5}\right), 139.7\left(\mathrm{~d},{ }^{3} \mathrm{~J}_{\mathrm{CP}}=13.8 \mathrm{~Hz}, \mathrm{C}_{1}{ }^{5}\right), 151.6\left(\mathrm{~d},{ }^{3} \mathrm{~J}_{\mathrm{CP}}=6.8 \mathrm{~Hz}, \mathrm{C}_{1}{ }^{1}\right), 152.0(\mathrm{~s}$, $\mathrm{C}_{0}^{1}$ ), 154.3 (s, NCOO), 155.2 (d, ${ }^{3} \mathrm{~J}_{\mathrm{CP}}=7.3 \mathrm{~Hz}, \mathrm{C}_{2}{ }^{1}$ ), 190.8 (s, CHO). MS (FAB) m/z: 1287 $[\mathrm{MH}]^{+}$. Anal. Calcd for $\mathrm{C}_{60} \mathrm{H}_{57} \mathrm{~N}_{8} \mathrm{O}_{13} \mathrm{P}_{3} \mathrm{~S}_{3}$ (1287.3): C, 55.98; H, 4.46; N, 8.70. Found: C, 55.69; H, 4.19; N, 8.41.

\section{Acknowledgements}

Thanks are due to the CNRS for financial support.

\section{References}

1. For reviews concerning dendrimers, see for example: (a) Tomalia, D. A.; Naylor, A. M.; Goddard, W. A. III Angew. Chem., Int. Ed. 1990, 29, 138. (b) Tomalia, D. A.; Durst, H. D. In Topics in Current Chemistry, Supramolecular Chemistry I: Directed Synthesis and Molecular Recognition, Weber, E., Ed. Springer-Verlag: Berlin, Heidelberg, 1993; Vol. 165, pp 193-313. (c) Issberner, J.; Moors, R.; Vögtle, F. Angew. Chem., Int. Ed. 1994, 33, 2413. (d) Newkome, G. R.; Moorefield, C. N.; Vögtle, F. In Dendritic Molecules: Concepts, Synthesis, Perspectives; VCH: Weinheim, 1996. (e) Fréchet J. M. J. Comprehensive Polym. Sci. 1996, 2, 71. (f) Gudat, D. Angew. Chem., Int. Ed. 1997, 36, 1951. (g) Chow, H. F.; Mong, T.K.K.; Nongrum, M. F.; Wan, C.W. Tetrahedron 1998, 54, 8543. (h) Matthews, O.A.; Shipway, A.N.; Stoddart, J.F. Prog. Polym. Sci. 1998, 23, 1. (i) Frey, H.; Lach, C.; Lorenz, K. Adv. Mater. 1998, 10, 279. (j) Gorman, C. Adv. Mater. 1998, 10, 295. (k) 
Fischer, M.; Vögtle, F. Angew. Chem. Int. Ed. 1999, 38, 884. (l) Majoral, J. P.; Caminade, A. M. Chem. Rev. 1999, 99, 845. (m) Bosman, A. W.; Janssen, H. M.; Meijer, E. W. Chem. Rev. 1999, 99, 1665. (n) Bosman, A. W.; Janssen, H.M.; Meijer, E.W. Chem. Rev. 1999, 99, 1665. (o) Newkome, G. R.; He, E.; Moorefield, C.N. Chem. Rev. 1999, 99, 1689. (p) Cuadrado, I.; Moran, M.; Casado, C. M.; Alonso, B.; Losada, J. Coord. Chem. Rev. 1999, 193-195, 395. (q) Archut, A.; Vögtle, F. Chem. Soc. Rev. 1999, 27, 233. (r) Hecht, S.; Fréchet, J. M. J. Angew. Chem., Int. Ed. 2001, 40, 75. (s) Oosterom, G. E.; Reek, J. N. H.; Kamer, P. C. J.; van Leeuwen, P. W. N. M. Angew. Chem. Int. Ed. 2001, 40, 1829. (t) Astruc, D.; Chardac, F. Chem. Rev. 2001, 101, 2991. (u) Advances in Dendritic Macromolecules Vol. 1; Newkome, G. R., JAI Press; 1994; pp 1-198. (v) Advances in Dendritic Macromolecules Vol. 2; Newkome, G.R., JAI Press; 1995; pp 1-204. (w) Advances in Dendritic Macromolecules Vol. 3; Newkome, G.R., JAI Press; 1996; pp 1-201. (x) Advances in Dendritic Macromolecules Vol. 4; Newkome, G.R., JAI Press; 1999; pp 1201.

2. (a) Buhleier, E.; Wehner, W.; Vögtle, F. Synthesis 1978, 155. (b) Tomalia, D. A.; Baker, H.; Dewald, J. R.; Hall, M.; Kallos, G.; Martin, S.; Roeck, J.; Ryder, J.; Smith, P. Polym. J. 1985, 17, 117. (c) Newkome, G. R.; Yao, Z.; Baker, G. R.; Gupta, V. K. J. Org. Chem. 1985, 50, 2003.

3. Hawker, C. J.; Fréchet, J. M. J. Macromolecules, 1990, 23, 4726.

4. (a) Launay, N.; Caminade, A. M.; Lahana, R.; Majoral, J. P. Angew. Chem., Int. Ed. 1994, 33, 1589. (b) Launay, N.; Caminade, A. M.; Majoral, J. P. J. Am. Chem. Soc. 1995, 117, 3282. (c) Galliot, C.; Prévoté, D.; Caminade, A.M.; Majoral, J. P. J. Am. Chem. Soc. 1995, 117, 5470. (d) Lartigue, M. L.; Donnadieu, B.; Galliot, C.; Caminade, A. M.; Majoral, J. P.; Fayet, J. P. Macromolecules 1997, 30, 7335. (e) Brauge, L.; Magro, G.; Caminade, A. M.; Majoral, J. P. J. Am. Chem. Soc. 2001, 123, 6698 (correction: J. Am. Chem. Soc. 2001, 123, 8446). (f) Merino, S.; Brauge, L.; Caminade, A. M.; Majoral, J. P.; Taton, D.; Gnanou, Y. Chem. Eur. J. 2001, 7, 3095.

5. See for example: (a) Slany, M.; Bardají, M.; Casanove, M. J.; Caminade, A. M.; Majoral, J. P.; Chaudret, B. J. Am. Chem. Soc. 1995, 117, 9764. (b) Lartigue, M. L.; Slany, M.; Caminade, A. M.; Majoral, J. P. Chem. Eur. J. 1996, 2, 1417. (c) Bardají, M.; Kustos, M.; Caminade, A. M.; Majoral, J. P.; Chaudret, B. Organometallics 1997, 16, 403. (d) Bardaji, M.; Caminade, A. M.; Majoral, J. P.; Chaudret, B. Organometallics 1997, 16, 3489. (e) Majoral, J. P.; Caminade, A. M. Topics in Current Chem. 1998, 197, 79. (f) Caminade, A. M.; Laurent, R.; Chaudret, B.; Majoral, J. P. Coord. Chem. Rev. 1998, 178-180, 793. (g) Loup, C.; Zanta, M. A.; Caminade, A. M.; Majoral, J. P.; Meunier, B. Chem. Eur. J. 1999, 5, 3644. (h) Schmid, G.; Meyer-Zaika, W.; Pugin, R.; Sawitowski, T.; Majoral, J. P.; Caminade, A. M.; Turrin, C. O. Chem. Eur. J. 2000, 6, 1693. (i) Turrin, C. O.; Chiffre, J.; de Montauzon, D.; Daran, J.C.; Caminade, A. M.; Manoury, E.; Balavoine, G.; Majoral, J. P. Macromolecules, 2000, 33, 7328. (j) Boggiano, M. K.; Soler-Illia, G. J. A. A.; Rozes, L.; Sanchez, C.; Turrin, C. O.; Caminade, A. M.; Majoral, J. P. Angew. Chem. Int. Ed. 2000, 39, 
4249. (k) Le Derf, F.; Levillain, E.; Gorgues, A.; Sallé, M.; Sebastian, R. M.; Caminade, A. M.; Majoral, J. P. Angew. Chem. Int. Ed. 2001, 40, 224. (l) Marmillon, C.; Gauffre, F.; Gulik-Krzywicki, T.; Loup, C.; Caminade, A. M.; Majoral, J. P.; Vors, J. P.; Rump, E. Angew. Chem. Int. Ed. 2001, 40, 2626.

6. (a) Larré, C.; Caminade, A. M.; Majoral, J. P. Angew. Chem., Int. Ed. 1997, 36, 596. (b) Galliot, C.; Larré, C.; Caminade, A. M.; Majoral, J. P. Science 1997, 277, 1981. (c) Larré, C.; Donnadieu, B.; Caminade, A. M.; Majoral, J. P. J. Am. Chem. Soc. 1998, 120, 4029. (d) Larré, C.; Donnadieu, B.; Caminade, A. M.; Majoral, J. P. Chem. Eur. J. 1998, 4, 2031. (e) Larré, C.; Bressolles, D.; Turrin, C.; Donnadieu, B.; Caminade, A. M.; Majoral, J. P. J. Am. Chem. Soc. 1998, 120, 13070. (f) Majoral, J. P.; Larré, C.; Laurent, R.; Caminade, A. M. Coord. Chem. Rev. 1999, 190-192, 3.

7. (a) Maraval, V.; Laurent, R.; Donnadieu, B.; Mauzac, M.; Caminade, A. M.; Majoral, J. P. J. Am. Chem. Soc. 2000, 122, 2499. (b) Maraval, V.; Prévôté-Pinet, D.; Laurent, R.; Caminade, A. M.; Majoral, J. P. New J. Chem. 2000, 24, 561. (c) Maraval, V.; Laurent, R.; Caminade, A. M.; Majoral, J. P. Organometallics 2000, 19, 4025. (d) Maraval, V.; Laurent, R.; Merino, S.; Caminade, A. M.; Majoral, J. P. Eur. J. Org. Chem. 2000, 3555. (e) Turrin, C. O.; Maraval, V.; Caminade, A. M.; Majoral, J. P.; Mehdi, A.; Reyé, C. Chem. Mat. 2000, 12, 3848.

8. $\quad$ Kunesch, N.; Miet, C.; Poisson, J. Tetrahedron Lett. 1987, 28, 3569. 